INSTITUT NATIONAL DE RECHERCHE EN INFORMATIQUE ET EN AUTOMATIQUE

\title{
Flow Control as Stochastic Optimal Control Problem with Incomplete Information
}

B. Miller - K. Avrachenkov - K. Stepanyan — G. Miller

$\mathbf{N}^{\circ} 5239$

June 2004

Thème COM 



\title{
Flow Control as Stochastic Optimal Control Problem with Incomplete Information
}

\author{
B. Miller* , K. Avrachenkov ${ }^{\dagger}$, K. Stepanyan ${ }^{\ddagger}$, G. Miller ${ }^{\S}$ \\ Thème COM — Systèmes communicants \\ Projet MAESTRO
}

Rapport de recherche $n^{\circ} 5239$ - June $2004-26$ pages

\begin{abstract}
The nonlinear stochastic control problem related with flow control is considered. The state of the link is described by controlled hidden Markov process while the loss flow is described by the counting process with the intensity depending on the current transmission rate and unobserved link state. The control is the transmission rate and have to be chosen as non-anticipating process depending on the observation of the loss process. The aim of the control is to achieve the maximum of some utility function taking into account the losses of transmitted information. Originally the problem belongs to a class of stochastic control with incomplete information, however, the optimal filtering equations giving the estimation of the current link state based on the observation of the loss process give the opportunity to reduce the problem to the standard stochastic control problem with full observations. Then, a necessary optimality condition is derived in the form of stochastic maximum principle which allows us to obtain explicit analytic expressions for the optimal control in some particular cases. The optimal and suboptimal controls are investigated and compared with the flow control schemes which is used in TCP/IP networks. In particular, the optimal control demonstrates a much smoother behaviour than the currently used TCP/IP congestion control.
\end{abstract}

Key-words: Flow Control, Optimal Stochastic Control, Incomplete Information, Filtering, Hidden Markov Models, TCP/IP

\footnotetext{
* Institute for Information Transmission Problem, 19 B. Karetny per., GSP-4, Moscow, 127994, Russia, e-mail: bmiller@iitp.ru.

$\dagger$ INRIA Sophia Antipolis, 2004, Route des Lucioles, B.P.93, 06902, France, e-mail: k.avrachenkov@sophia.inria.fr.

$\ddagger$ Institute for Information Transmission Problem, 19 B. Karetny per., GSP-4, Moscow, 127994, Russia, e-mail: kstepanyan@iitp.ru.

$\S$ Moscow State Aviation Institute, 4 Volokolamskoe Shosse, GSP-3, 125993, Moscow, Russia, e-mail: gmiller@ipiran.ru.
} 


\section{Le Contrôle de Flux vu comme un Problème de Commande Optimale Stochastique avec Information Partielle}

Résumé : Nous étudions le contrôle de flux des données dans le contexte de la théorie de commande optimale stochastique avec information partielle. Le lien de transmission est modelisé par une chaîne de Markov cachée. Le contrôle est le taux de la transmission qui doit etre choisi comme un processus prévisible dépendant de l'observation du processus de pertes. Le but du contrôle est maximiser une combinaison lináire d'une fonction d'utilité et du coût de pertes. Grace au filtrage optimal d'état du lien, nous pouvons transformer ce problème avec information partielle en un problème avec information complete. Une condition nécessaire d'otimalié est obtenue sous forme du principe de maximum stochastique. Cette condition nous permet de déveloper des expressions analytiques pour le contrôle optimal dans quelques cas particulières. Finalement, les contrôles optimaux et sous-optimaux (correspondants aux versions courantes de TCP/IP) sont comparés. En particulier, nous montrons que le contrôle optimal est plus lisse que le mécanisme du contrôle de flux utilisé par la version standard de TCP/IP.

Mots-clés : Contrôle de Flux, Commande Optimale Stochastique, Information Partielle, Filtrage, Modèles de Markov cachés, TCP/IP 


\section{Introduction}

The transmission of most Internet data flows is governed by TCP (Transmission Control Protocol) $[15,1]$. TCP has two principal aims: to ensure reliable data delivery and to control congestion level in the network. The TCP New Reno version, which is currently the most widely deployed TCP version, uses either packet losses or ECN (Explicit Congestion Notification, [28]) marks as an indication of the network congestion. Congestion Avoidance, the main TCP phase, corresponds to AIMD (Additive Increase Multiple Decrease) congestion control scheme with a binary feedback [27]. The evolution of the TCP sending rate can be viewed as a piece-wise deterministic process $[2,3,6]$. Namely, TCP increases the data sending rate linearly in time in the absence of congestion notifications and when a congestion notification arrives, TCP reduces the sending rate by half. Up to the present, several fundamental questions about TCP properties have not been answered. These questions are: What are the reasons behind the piece-wise deterministic TCP sending rate evolution? What should be an optimal increase rate in the absence of congestion notifications? Is it optimal do divide the sending rate by two if a congestion notification arrives? In this paper we provide answers to these questions from the point of view of stochastic optimal control with incomplete information $[8,10,11]$. Specifically, we describe a congested link as a Hidden Markov Model [10]. We note that a commonly used Gilbert link model [14] is a particular instance of Hidden Markov Models. As an optimization goal we choose to maximize the average utility function of the data sending rate minus a cost of transfer (or a cost of lost packets). Each congestion notification contributes some fixed amount to the cost of transfer. The present optimization criterion resembles the optimization criteria of $[5,17,18,19,21,23]$. However, the models in these references are fluid models which are derived to calculate an average TCP sending rate and hence cannot be used to analyze the behavior of the instantaneous TCP sending rate. In fact, comparing [5], [21] and [18] one can see that different transmission rate control schemes can be proposed which solve the same optimization problem. The dependence of the cost counting process on the transmission rate makes our problem especially difficult as the intensity of jumps of the sending rate becomes dependent on the sending rate itself. Note that in $[2,3]$ the intensity of jumps of the corresponding piece-wise deterministic process is independent of the sending rate. Only in [29] some asymptotics have been obtained for the piece-wise deterministic Markov process with rate dependent jumps for small intensity of congestion notifications. However, [29] provides the performance evaluation of the current TCP New Reno and does not address the question of the optimal design of the congestion control mechanism. The present work explains the reasons for the piece-wise deterministic form of the sending rate evolution and provides means to set an optimal time-varying rate of the sending rate increase and an optimal size of the downward jumps.

The paper is organized as follows: In the next Section 2, we present the Hidden Markov Model of the congested path and the performance criterion. In Section 3, using the sufficient statistics set, we transform the originally incomplete information problem to the problem of the optimal control based on complete observations. In particular, we give the characterization of the optimal control problem solution in terms of piece-wise deterministic Markov

$\mathrm{RR} \mathrm{n}^{\circ} 5239$ 
control settings. In Section 4 we consider a further transformation of the problem with the aid of Girsanov's change of measure. The latter allows us to provide the characterization of the optimal control in terms of necessary optimality conditions. In particular, these conditions give the possibility to calculate the optimal control if the generator of the underlying Hidden Markov Model does not depend on the control. Then, in Section 5 by means of a simple numerical example we compare the optimal control with the "suboptimal" solution corresponding to the AIMD congestion avoidance scheme. We recall that AIMD is the principle phase of the current TCP New Reno version. Remarkably, the optimal control demonstrates a much smoother behavior than the AIMD congestion avoidance scheme. The paper is concluded in Section 6, where we discuss future possible developments of this research direction.

\section{Model description and the problem statement}

\section{Hidden Markov Model of the congested path}

Suppose that all processes are defined on a probability space $\{\Omega, \mathcal{F}, P\}$. We assume that the state of a congested path in the Internet can be described by a Markov process $X_{t}$, whose state space is the set of unit vectors $\left\{e_{1}, \ldots, e_{n}\right\}$ of $R^{n}$. Write $p_{t}^{i}=P\left(X_{t}=e_{i}\right)$ and suppose there is a time-dependent family of generators $A^{*}(t, u)^{1}$ which also depends on the scalar control parameter $u \in U=\left[0, U_{\max }\right], \quad U_{\max }<\infty$. The control parameter $u$ corresponds to current value of the transmission rate, such that the probability vector $p_{t}=\left\{p_{t}^{1}, \ldots, p_{t}^{n}\right\}^{*}$ satisfies the Kolmogorov forward equations

$$
\frac{d p_{t}}{d t}=A\left(t, u_{t}\right) p_{t}
$$

We assume that matrix valued function $A(t, u)$ is continuous with respect to both variables, so the above equation has a unique solution for any measurable admissible control, such that $u_{t} \in U$, a.s..

Suppose that one can observe the binary congestion notification feedback which is correlated with the current state of the link $X_{t}$ and the current value of the data sending rate $u_{t}$. We recall that the data is sent in packets in the Internet. To indicate the level of congestion the network sends the flow of congestion notifications back to a source. The congestion notifications in the Internet are either packet losses or ECN marks. To represent the flow of congestion notifications we use the generalized counting Poisson process $N_{t}$ with the intensity

$$
c\left(X_{t}\right) u_{t}=<c, X_{t}>u_{t},
$$

where $\langle\cdot, \cdot\rangle$ is a scalar product, and $c=\left\{c\left(e_{1}\right), \ldots, c\left(e_{n}\right)\right\}^{*}$ is such that $c\left(e_{i}\right)>0$, for all $e_{i}=1, \ldots, n$.

\footnotetext{
${ }^{1}$ Here and below $A^{*}$ denotes the transposed matrix $A$.
} 
We note that $\left\{X_{t}, N_{t}\right\}$ is a controlled jump Markov process with countable state space. The control $u_{t}$ belongs to $D_{[0, T]}(U)$, the set of functions continuous from the left and with a finite limit from the right. In particular, we have

$$
\begin{aligned}
& P\left\{X_{t+\Delta t}=e_{i}, N_{t+\Delta t}=j \mid X_{t}=e_{k}, N_{t}=l, u_{t}^{t+\Delta t}(\cdot)\right\}= \\
& \left\{\begin{array}{l}
o(\Delta t), \quad \text { if } \quad i \neq k, j \neq l ; \\
a_{i k}\left(t, u_{t}\right) \Delta t+o(\Delta t), \quad \text { if } \quad i \neq k, j=l ; \\
c\left(e_{i}\right) u_{t} \Delta t+o(\Delta t), \quad \text { if } \quad i=k, j=l+1 ; \\
1-\left[\sum_{k \neq i} a_{i k}\left(t, u_{t}\right)+c\left(e_{i}\right) u_{t}\right] \Delta t+o(\Delta t), \quad \text { if } i=k, j=l,
\end{array}\right.
\end{aligned}
$$

with

$$
o(\Delta t) / \Delta t \rightarrow 0, \quad \text { as } \quad \Delta t \rightarrow 0,
$$

uniformly with respect to $(t, u, i, j, k, l)$ and where $a_{i k}\left(t, u_{t}\right)$ is the $i k$-element of matrix $A(t, u)$. We would like to emphasize that the intensity of $N_{t}$ depends linearly on the control $u_{t}$. In particular, this assumption is justified if for some fixed state of the underlying Hidden Markov Model either packets are lost independently with some probability [29] or ECN marks are sent by Random Early Detection routers with linear marking function [12].

Next, let us introduce a semimartingale representation of the above Markov jump process $[7,20]$. Towards this goal, let us first introduce the following right-continuous sets of complete $\sigma$-algebras generated by $X_{t}$ and $N_{t}$, respectively:

$$
\mathcal{F}_{t}^{X}=\sigma\left\{X_{s}: s \in[0, t]\right\} \quad \mathcal{F}_{t}^{N}=\sigma\left\{N_{s}: s \in[0, t]\right\} \quad \text { and } \quad \mathcal{F}_{t}^{X, N}=\mathcal{F}_{t}^{X} \vee \mathcal{F}_{t}^{N} .
$$

We suppose that the set of admissible control functions $\{u\}$ is the set of $\mathcal{F}_{t}^{N}$-predictable processes with values in $U$. It means that $N_{t}$ is the number of jumps occurred until the current time $t$ and if $\left\{\tau_{1}, \ldots, \tau_{N_{t}}\right\}$ are the jump times of $N$, then for $\tau_{N_{t}}<t \leq \tau_{N_{t}+1}$ control $u_{t}=u\left(t, N_{0}^{t}\right)$ is a function of $\left\{\tau_{1}, \ldots, \tau_{N_{t}}\right\}$ and current time $t[10]$. Then, the process $\left\{X_{t}, N_{t}\right\}$ admits the following semimartingale representation

$$
\begin{gathered}
X_{t}=X_{0}+\int_{0}^{t} A\left(s, u_{s}\right) X_{s} d s+M_{t}, \\
N_{t}=\int_{0}^{t}<c, X_{s}>u_{s} d s+\nu_{t},
\end{gathered}
$$


where $X_{0}$ is the initial state of the path, and $M_{t}:=\left\{M_{t}^{1}, \cdots, M_{t}^{n}\right\}, \nu_{t}$ are square integrable $\left(\mathcal{F}_{t}^{X, N}, P\right)$ martingales with the following quadratic variations

$$
\begin{gathered}
\langle M\rangle_{t}=-\int_{0}^{t}\left[A\left(s, u_{s}\right)\left(\operatorname{diag} X_{s}\right)+\left(\operatorname{diag} X_{s}\right) A^{*}\left(s, u_{s}\right)\right] d s \\
+\int_{0}^{t}\left(\operatorname{diag} A\left(s, u_{s}\right) X_{s}\right) d s, \\
\langle\nu\rangle_{t}=\int_{0}^{t}\left\langle c, X_{s}>u_{s} d s,\right. \\
\langle\nu, M\rangle_{t}=0,
\end{gathered}
$$

where $\operatorname{diag} X$ denotes the matrix with diagonal entries $X^{1}, \ldots, X^{n}$. The equation (6) takes place, since the processes $X_{t}$ and $N_{t}$ are pure jump processes and due to (1) with probability one they do not jump at the same time moment.

We would like to note that even though the above Hidden Markov Model has some similarity to the congested path model proposed in [3,4], an important difference between two approaches is that here the rate of congestion notifications depends on the data sending rate.

\section{Performance criterion}

The optimization goal is to maximize the time average utility function of the data sending rate minus a cost of transfer. Each congestion notification contributes some fixed amount to the cost of transfer. Let $T$ be the duration of data transfer. Then, the performance criterion can be written as follows:

$$
J[U(\cdot)]=E\left\{\int_{0}^{T} f_{0}\left(u_{s}, X_{s}\right) d s-k N_{T}\right\},
$$

with

$$
f_{0}(u, x)=<f_{0}(u), x>
$$

where $f_{0}^{*}(u)=\left(f_{0}\left(u, e_{1}\right), \ldots, f_{0}\left(u, e_{n}\right)\right)$, and each $f_{0}\left(\cdot, e_{i}\right)$ is a utility function of sending rate when the congested path is in state $e_{i}$. It is increasing and concave and satisfies the following assumption

$$
\lim _{u \rightarrow \infty}\left[f_{0}\left(u, e_{i}\right)-c u\right]=-\infty
$$

for any $c>0, e_{i}, i=1, \ldots, n$.

The parameter $k$ can be interpreted either as a cost of information loss or $k c\left(e_{i}\right)$ as a price of bandwidth when the congested path is in state $e_{i}$ [21]. 
Remark 1 We would like to note that condition (8) allows to consider control from some unbounded set $U$. Indeed, since for any $i=1, \ldots, n$ and given $k$ the function $f_{0}\left(u, e_{i}\right)-k c\left(e_{i}\right) u$ is uniformly bounded, there exists $u_{\text {max }}^{i}$ such that

$$
f_{0}\left(u, e_{i}\right)-k c\left(e_{i}\right) u \leq f_{0}\left(u_{\max }^{i}, e_{i}\right)-k c\left(e_{i}\right) u_{\max }^{i}
$$

for $u \geq u_{\max }^{i}$. Let $U_{\max }=\max _{i} u_{\max }^{i}$. Thus, if we take the modified uniform bounded control

$$
\tilde{u}_{t}=\left\{\begin{array}{c}
u_{t}, \quad \text { if } u_{t} \leq U_{\max }, \\
U_{\max }, \quad \text { if } u_{t}>U_{\max }
\end{array}\right.
$$

instead of unbounded control $u_{t}$, the value of the performance criterion cannot be decreased.

We would like to emphasize that we obtain the indirect information concerning the link state through the loss process only. We cannot measure the state dependent loss process intensity directly, so we are coming to an instance of the joint process-observation problems [25].

\section{$3 \quad$ Filtering equations and the problem reduction}

Let us transform the stochastic optimal control problem (2)-(7) with incomplete information to a standard stochastic optimal control problem within the class of completely observable Markov processes. This transformation can be done by using the set of sufficient statistics

$$
\pi_{t}^{i}=E\left\{X_{t}^{i} \mid \mathcal{F}_{t}^{N}\right\}, \quad i=1, \ldots, n .
$$

Theorem 1 Suppose that $u_{s}$ is $\mathcal{F}_{t}^{N}$ - predictable control, then the components of vector

$$
\pi_{t} \in R^{n}, \text { where } \pi_{t}=\left\{\pi_{t}^{1}, \ldots, \pi_{t}^{n}\right\}^{*}
$$

satisfy the following system of stochastic differential equations

$$
d \pi_{t}=A\left(t, u_{t}\right) \pi_{t} d t+\Gamma\left(\pi_{t-}\right) d \tilde{\nu}_{t},
$$

where

$$
\tilde{\nu}_{t}=N_{t}-\int_{0}^{t} \sum_{i=1}^{n} \pi_{s}^{i} c\left(e_{i}\right) u_{s} d s=N_{t}-\int_{0}^{t}<\pi_{s}, c>u_{s} d s,
$$

is a square integrable martingale with quadratic variation

$$
\langle\tilde{\nu}\rangle_{t}=\int_{0}^{t}<\pi_{s}, c>u_{s} d s
$$


and

$$
\Gamma(\pi)=\frac{1}{\sum_{i=1}^{n} \pi^{i} c\left(e_{i}\right)}\left(\begin{array}{c}
\pi^{1} c\left(e_{1}\right) \\
\cdots \cdots \\
\pi^{n} c\left(e_{n}\right)
\end{array}\right)-\left(\begin{array}{c}
\pi^{1} \\
\cdots \\
\pi^{n}
\end{array}\right) .
$$

At the same time the criterion can be expressed as follows

$$
J[u(\cdot)]=E\left\{\int_{0}^{T}<\pi_{s}, f_{0}\left(u_{s}\right)-k c u_{s}>d s\right\} .
$$

Proof: For any $\mathcal{F}_{t}^{X, N}-$ measurable process $h(t)$, denote $\hat{h}_{t}=E\left\{h(t) \mid \mathcal{F}_{t}^{N}\right\}$. Then $\pi_{t}=\hat{X}_{t}$. As follows from (2)

$$
\hat{X}_{t}=X_{0}+\int_{0}^{t} A\left(s, u_{s}\right) \hat{X}_{s} d s+\hat{M}_{t}
$$

where $\hat{M}_{t}$ is a square integrable $\mathcal{F}_{t}^{N}$ martingale, which therefore admits the representation $[10],[20]$

$$
\hat{M}_{t}=\int_{0}^{t} \gamma_{s} d \tilde{\nu}_{s}
$$

where $\tilde{\nu}_{s}$ is the innovation process

$$
\tilde{\nu}_{s}=N_{t}-\int_{0}^{t}<c, \hat{X}_{s}>u_{s} d s
$$

and $\gamma_{s}$ is some square integrable process. By using the Ito formula one can calculate the product $X_{t} N_{t}$, which gives

$$
X_{t} N_{t}=\int_{0}^{t} A\left(s, u_{s}\right) X_{s} N_{s} d s+\int_{0}^{t} N_{s} d M_{s}+\int_{0}^{t} X_{s-}<c, X_{s}>u_{s} d s+<M, \nu>_{t},
$$

where $\langle M, \nu\rangle_{t}$ is a mutual quadratic variation of martingales $M, \nu$ and since the process $X$ has no jumps in common with $N$ then $\langle M, \nu\rangle_{t}=0$. Taking the conditional expectation and by using Wang and Hajek result (Lemma 3.2 p. 261 [31]) we obtain

$$
\hat{X}_{t} N_{t}=\int_{0}^{t} A\left(s, u_{s}\right) \hat{X}_{s} N_{s} d s+\int_{0}^{t} X_{s-} \widehat{<c, X}_{s-}>u_{s} d s+\text { martingale. }
$$


Then by calculating the same expression with the aid of the Ito formula taking into account the representation (13) we obtain

$$
\begin{aligned}
\hat{X}_{t} N_{t}= & \int_{0}^{t} A\left(s, u_{s}\right) \hat{X}_{s} N_{s} d s+\int_{0}^{t} \hat{X}_{s-}<c, \hat{X}_{s-}>u_{s} d s+ \\
& \int_{0}^{t} \gamma_{s}<c, \hat{X}_{s-}>u_{s} d s+\text { martingale. }
\end{aligned}
$$

By equating the predictable processes of bounded variation in (14) and (15) we yield the equation

$$
\int_{0}^{t} X_{s-}{\widehat{<c, X_{s-}}}^{t} u_{s} d s=\int_{0}^{t} \hat{X}_{s-}<c, \hat{X}_{s-}>u_{s} d s+\int_{0}^{t} \gamma_{s}<c, \hat{X}_{s-}>u_{s} d s .
$$

Since $<c, \hat{X}_{s-}>>0$ we obtain, finally the expression

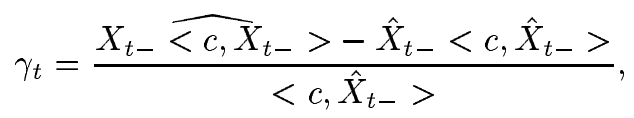

which coincides with (11).

The expression (12) for criterion function is a straightforward consequence of the same Wang and Hajek form of Fubbini theorem (Lemma 3.2 p. 261 [31]) and representation of $N_{t}$ by expression (3)

$$
\begin{gathered}
J[U(\cdot)]=E\left\{\int_{0}^{T} f_{0}\left(u_{s}, X_{s}\right) d s-k N_{T}\right\}= \\
E\left\{\int_{0}^{T} E\left\{f_{0}\left(u_{s}, X_{s}\right)-k<c, X_{s}>u_{s} \mid \mathcal{F}_{s}^{N}\right\} d s\right\}= \\
E\left\{\int_{0}^{T}<\pi_{s}, f_{0}\left(u_{s}\right)-k c u_{s}>d s\right\} .
\end{gathered}
$$

First observation of the problem (10)-(12) shows that it belongs to a class of piece-wise deterministic control settings [8] since the evolution of the state process $\pi_{t}$ is deterministic between jumps, and the jumps occur with intensity

$$
\lambda_{t}^{u}=\sum_{i=1}^{n} \pi_{t}^{i} c\left(e_{i}\right) u_{t}=<\pi_{t}, c>u_{t} .
$$

Moreover, the jumps in itself are deterministic and described by relation

$$
\Delta \pi_{\tau}=\Gamma\left(\pi_{\tau-}\right) .
$$


The dynamical programming approach gives the following characterization of the price. Define a function

$$
V(x, t)=\sup _{u(\cdot)} J\left[u(\cdot) \mid \pi_{t}=x\right],
$$

where supremum should be taken over the set of $\mathcal{F}_{t}^{N}$ - predictable controls, and

$$
J\left[u(\cdot) \mid \pi_{t}=x\right]=E\left\{\int_{t}^{T}\left[\sum_{i=1}^{n} \pi_{s}^{i}\left(f_{0}\left(u_{s}, e_{i}\right)-k c\left(e_{i}\right) u_{s}\right)\right] d s\right\}
$$

conditioned by $\pi_{t}=x$. Define the equation

$$
\begin{gathered}
0=V_{t}^{\prime}(x, t)+\sup _{u \in U}\left\{V_{x}^{\prime}(x, t)[A(t, u) x-\Gamma(x)<c, x>u]\right. \\
\left.+[V(x+\Gamma(x), t)-V(x, t)]<c, x>u+<f_{0}(u)-k c u, x>\right\}
\end{gathered}
$$

with terminal condition $V(x, T)=0$.

Theorem 2 Assume that:

1. $U$ is a compact set;

2. $V$ is a piece-wise $C^{1}$ solution of (17) on

$$
E=\left\{x: x^{i} \geq 0, \sum_{i=1}^{n} x^{i}=1\right\} \times[0, T]
$$

3. There exist $\hat{u}(x, t) \in U$, such that at each $(x, t) \in E$ the value in r.h.s. of (17) achieves the maximum at $u_{0}=\hat{u}(x, t)$.

4. There exist a unique solution of equation

$$
d x_{t}=A\left(t, \hat{u}\left(x_{t}, t\right)\right) x_{t} d t+\Gamma\left(x_{t-}\right) d \tilde{\nu}_{t}^{\hat{u}} .
$$

Then $\hat{u}(x, t)$ is the optimal control and $V(x, t)=J[\hat{u}]$.

Proof: The idea of demonstration consists in standard application of Ito's formula provided by the uniform boundness of $\left\langle\tilde{\nu}^{u}\right\rangle_{T}$ [8], [11]. Take an arbitrary admissible control $u_{s}$ and the corresponding solution $x_{s}^{u}$, such that $x_{t}=x$. Then $V\left(x_{T}^{u}, T\right)=0$, and by Ito's formula we have

$$
\begin{gathered}
0=V(x, t)+\int_{t}^{T} V_{x}^{\prime}\left(x_{s}, s\right)\left\{A\left(s, u_{s}\right) x_{s-}^{u}-\Gamma\left(x_{s-}^{u}\right)<c, x_{s-}^{u}>u_{s}+\right. \\
\left.\left[V\left(x_{s-}^{u}+\Gamma\left(x_{s-}^{u}\right)\right)-V\left(x_{s-}^{u}\right)\right]<c, x_{s-}^{u}>u_{s}\right\} d s+ \\
\int_{t}^{T}\left[V\left(x_{s-}^{u}+\Gamma\left(x_{s-}^{u}\right)\right)-V\left(x_{s-}^{u}\right)\right] d \tilde{\nu}_{s} .
\end{gathered}
$$

INRIA 
Add to both sides of (18) the value of

$$
\int_{t}^{T}<f_{0}\left(u_{s}\right)-k c u_{s}, x_{s}^{u}>d s
$$

and take the expectation. Since the solution $x_{s}^{u} \in E$ is bounded and $V$ is continuous, the expectation of the bounded function over the martingale $\tilde{\nu}_{s}$ with uniformly bounded variation (recall that $U$ is a compact set) is equal to zero and taking into account (17) we obtain

$$
J[u(\cdot)]=E\left\{\int_{t}^{T}<f_{0}\left(u_{s}\right)-k c u_{s}, x_{s}^{u}>d s\right\} \leq V(x, t) .
$$

However the substitution of control $\hat{u}\left(x_{s}, s\right)$ yields the equality, which completes the proof.

\section{Reduction of the problem with the aid of Girsanov's transformation and derivation of necessary optimality conditions}

As was noticed before, the dependence of the driving counting process on the control makes the present problem very difficult for the analysis. In particular, it is not evident to compare the efficiency of two different controls since they generate different flows of loss and therefore driving counting processes are different. It appears useful to search for the equivalent problem setting where the driving process is control independent. The Girsanov's measure transformation gives such opportunity. We are using here the reduction of the filtering problem in terms of unnormalized conditional distribution of $X_{t}^{u}$ [10]. Suppose that on a probability space $\left(\Omega, \mathcal{F}^{N}, \bar{P}\right), N_{t}, 0<t \leq T$ is a standard Poisson process and the observations are given by Markov process $X_{t}^{u}$ which satisfies equation (2) with some $\mathcal{F}^{N}$-predictable control $u_{s}>0, \mathrm{P}$-a.s. almost everywhere on $[0, T]$. Then $\bar{Q}_{t}=N_{t}-t$ is a $\mathcal{F}^{N}$-martingale under $\bar{P}$.

For any $\mathcal{F}^{N}$ - predictable control $u_{s}$ consider the family random variables

$$
\bar{\Lambda}_{t}^{u}=\left(\prod_{0<\tau \leq t} c\left(X_{\tau-}\right) u_{\tau-} \Delta N_{\tau}\right) \exp \left(\int_{0}^{t}\left(1-c\left(X_{\tau}\right)\right) u_{\tau} d \tau\right) .
$$

Then, it follows from the Ito's formula that $\bar{\Lambda}_{t}^{u}$ satisfies the next equation

$$
\bar{\Lambda}_{t}^{u}=1+\int_{0}^{t} \bar{\Lambda}_{\tau-}^{u}\left(c\left(X_{\tau-}\right) u_{\tau-}-1\right) d \bar{Q}_{\tau} .
$$


Since the control is positive, we have that $\Delta \bar{\Lambda}_{t}>-1$, P-a.s.. Furthermore, since the process $\left(c\left(X_{\tau_{-}}\right) u_{\tau_{-}}-1\right)$ is uniformly bounded, the solution of equation $(19)$ is a $\left(\mathcal{F}_{t}^{N}, \bar{P}\right)$ martingale. Next we define a new probability measure $P$ by

$$
\bar{E}\left[\frac{d P}{d \bar{P}} \mid \mathcal{F}_{t}^{N}\right]=\bar{\Lambda}_{t}^{u}
$$

Using the Abstract Girsanov's Theorem [10, Ch.13], [31, Ch.6], one can transform the semimartingale characteristics as specified in the next proposition.

Proposition 1 On the probability space $\left(\Omega, \mathcal{F}^{X, N}, P\right)$, the process defined by

$$
\nu_{t}=\bar{Q}_{t}+t-\int_{0}^{t} c\left(X_{\tau}\right) u_{\tau-} d \tau
$$

is a square integrable martingale with quadratic variation

$$
<\nu>_{t}=\int_{0}^{t} c\left(X_{\tau}\right) u_{\tau} d \tau, \quad P \text {-a.s. }
$$

Moreover, for any $\left\{t_{1}, t_{2}, \ldots, t_{k} \leq t\right\}$ and any Borel function $\phi$ the following relation holds

$$
E\left[\phi\left(X_{t_{1}}^{u}, \ldots, X_{t_{k}}^{u} ; \tilde{N}_{t_{1}}^{u}, \ldots, \tilde{N}_{t_{k}}^{u}\right]=\bar{E}\left[\bar{E}\left\{\bar{\Lambda}_{t}^{u} \phi\left(X_{t_{1}}^{u}, \ldots, X_{t_{k}}^{u} ; N_{t_{1}}, \ldots, N_{t_{k}}\right) \mid \mathcal{F}_{t}^{N}\right\}\right],\right.
$$

where the expectation $E$ is calculated with respect to the measure $P$, and the expectation $\bar{E}$ is calculated with respect to the measure $\bar{P}$.

Thus, Girsanov's transformation of measure allows us to construct a weak solution $\left(X_{t}^{u}, \tilde{N}_{t}^{u}\right)$ for the controlled system $(2),(3)$ on the probability space $\left(\Omega, \mathcal{F}^{X, N}, P\right)$. This weak solution coincides per trajectory with a strong solution $\left(X_{t}^{u}, N_{t}\right)$ on the probability space $\left(\Omega, \mathcal{F}^{X, N}, \bar{P}\right)$. In particular, by Proposition 1 , since measure $P$ is absolutely continuous with respect to measure $\bar{P}$, one can calculate the moments of the controlled process along the trajectories of the strong solution when the system is driven by a standard Poisson process. Therefore, we are able to formulate the following equivalent optimization problem.

Theorem 3 The unnormalized conditional distribution of $X_{t}$ given $N_{t}$ is then given by

$$
q_{t}^{u}=\bar{E}\left[\bar{\Lambda}_{t}^{u} X_{t} \mid \mathcal{F}_{t}^{N}\right]=q_{0}+\int_{0}^{t} A\left(s, u_{s}\right) q_{s} d s+\int_{0}^{t} B\left(u_{s-}\right) q_{s-} d \bar{Q}_{s}
$$

where

$$
B(u)=\operatorname{diag}(c) u-I,
$$


with $I$ as $n \times n$ identity matrix. The cost function is equal to

$$
J[u(\cdot)]=\bar{E} \int_{0}^{T}<f_{0}\left(u_{s}\right)-k c u_{s}, q_{s}^{u}>d s .
$$

Proof: First part of the theorem concerning the equation (20) follows immediately from Lemma 2.2 page 293 in [10].

Then, to transform the performance criterion, we use Proposition 1.

$$
\begin{gathered}
J[u(\cdot)]=E\left\{\int_{0}^{T} f_{0}\left(u_{s}, X_{s}^{u}\right) d s-k \tilde{N}_{T}^{u}\right\}= \\
\bar{E}\left\{\bar{E}\left[\bar{\Lambda}_{T}^{u} \int_{0}^{T} f_{0}\left(u_{s}, X_{s}\right) d s \mid \mathcal{F}_{T}^{N}\right]\right\}-k \bar{E}\left\{\bar{E}\left[\bar{\Lambda}_{T}^{u} N_{T} \mid \mathcal{F}_{T}^{N}\right]\right\}=J_{1}-J_{2} .
\end{gathered}
$$

Next, taking into account that $N_{t}$ and $\bar{\Lambda}_{t}^{u}$ are functions of bounded variation, we use the Ito's formula and the results of Wang and Hajek (Lemma 3.2, Chapter 7, [31]).

$$
\begin{aligned}
J_{1}= & \bar{E}\left\{\bar{E}\left[\int_{0}^{T} \bar{\Lambda}_{s-}^{u} f_{0}\left(u_{s}, X_{s}\right) d s+\int_{0}^{T}\left[\int_{0}^{s} f_{0}\left(u_{\tau}, X_{\tau}\right) d \tau\right] d \bar{\Lambda}_{s-}^{u} \mid \mathcal{F}_{T}^{N}\right]\right\}= \\
& \bar{E}\left\{\int_{0}^{T} \bar{E}\left[\bar{\Lambda}_{s-}^{u} f_{0}\left(u_{s}, X_{s}\right) \mid \mathcal{F}_{s}^{N}\right] d s\right\}=\bar{E}\left\{\int_{0}^{T}<f_{0}\left(u_{s}\right), q_{s}^{u}>d s\right\} .
\end{aligned}
$$

Next, using the representation $N_{t}=\bar{Q}_{t}-t$ for the Poisson process $N_{t}$, we obtain

$$
\begin{gathered}
k \bar{E}\left\{\bar{E}\left[\int_{0}^{T} \bar{\Lambda}_{s-}^{u}\left(d \bar{Q}_{s}+d s\right)+\int_{0}^{T} N_{s-} d \bar{\Lambda}_{s-}^{u}+\int_{0}^{T} \bar{\Lambda}_{s-}^{u}\left(c\left(X_{s}\right) u_{s-}-1\right) d s \mid \mathcal{F}_{T}^{N}\right]\right\}= \\
k \bar{E}\left\{\bar{E}\left[\int_{0}^{T} \bar{\Lambda}_{s-}^{u} c\left(X_{s}\right) u_{s-} d s \mid \mathcal{F}_{T}^{N}\right]\right\}=k \bar{E}\left\{\int_{0}^{T}\left\langle c u_{s-}, q_{s}^{u}\right\rangle d s\right\},
\end{gathered}
$$

which completes the proof.

Remark 2 The correspondence between $\pi_{t}$ and $q_{t}^{u}$ is specified by relations

$$
\pi_{t}=\frac{q_{t}^{u}}{\bar{\Lambda}_{t}^{u}}=\frac{q_{t}^{u}}{\sum_{i=1}^{n}\left(q_{t}^{u}\right)^{i}} .
$$

$\mathrm{RR} \mathrm{n}^{\circ} 5239$ 
Remark 3 Finally we obtain the new optimal control problem for system (20) with the cost function (21) which is equivalent to the original problem, but much more easy for implementation since the driving process does not depend on control and the equation (20) is linear in $q^{u}$.

Let us suppose that there exists a $\mathcal{F}^{N}$-predictable optimal control $u_{t}^{*}$ and derive the necessary optimality condition. There are well developed theory of necessary optimality conditions [16], [30] for control processes driven by Poisson type noises, however, in our case of linear dynamic one can obtain the optimality condition more easily by direct calculation of Gâteaux derivative of the performance criterion.

Define the function

$$
H(\psi, q, u, t)=<f_{0}(u)-k c u, q>+<\psi, A(t, u) q>.
$$

Theorem 4 Assume that $\left(u_{t}^{*}, q_{t}^{u^{*}}\right)$ is an optimal process in the problem (20), (21). Then there exists the $\mathcal{F}_{t}^{N}$ measurable, continuous and square integrable process $\psi_{t}$ satisfying the backward stochastic differential equation

$$
\begin{gathered}
d \psi_{t}=-\left[\operatorname{diag}(c) u_{t-} \Delta \bar{Q}_{t}+I\right]^{-1}\left\{A\left(t, u_{t}^{*}\right) d t+B\left(u_{t}^{*}\right) d \bar{Q}_{t}\right\}^{*} \psi_{t-}+ \\
\left(\Phi^{u^{*}}(t-, 0)\right)^{-1} \phi_{t} d \bar{Q}_{t}-\left[f_{0}\left(u_{t}^{*}\right)-k c u_{t}^{*}\right] d t
\end{gathered}
$$

with some square integrable $\mathcal{F}^{N}$ predictable process $\phi_{t}$ and with terminal condition $\psi_{T}=0$, such that optimal control satisfies the maximum condition

$$
H\left(\psi_{t}, q_{t}^{u^{*}}, u_{t}^{*}, t\right)=\max _{u \in U} H\left(\psi_{t}, q_{t}^{u^{*}}, u, t\right) .
$$

We anticipate the proof by the ensuing lemma. Notice that solution of (20) can be expressed as

$$
q_{t}^{u}=\Phi^{u}(t, 0) q_{0},
$$

where $\Phi^{u}$ is a fundamental solution of the linear equation

$$
d \Phi^{u}(t, s)=A\left(t, u_{t}^{*}\right) \Phi^{u}(t, s) d t+B\left(u_{t}^{*}\right) \Phi^{u}(t, s) d \bar{Q}_{t},
$$

with initial condition $\Phi^{u}(t, s)=I$, the $n \times n$ identity matrix. Define a process

$$
\psi_{t}=\bar{E}\left[\int_{t}^{T}\left(\Phi^{u^{*}}(s, t)\right)^{*}\left[f_{0}\left(u_{s}^{*}\right)-k c u_{s}^{*}\right] d s \mid \mathcal{F}_{\tau}^{N}\right],
$$

Lemma 1 For given control $u_{t}^{*}$ there exists a square integrable $\mathcal{F}^{N}$ predictable process $\phi_{t}$ such that $\psi_{t}$ (see (26)) satisfies the backward stochastic differential equation (23). 
Proof: For any given predictable bounded control $u_{t}$ consider the stochastic differential equation

$$
d \Theta(t, \tau)=-\Theta(t-, \tau)\left\{A\left(t, u_{t}\right) d t+B\left(u_{t}\right) d \bar{Q}_{t}\right\}\left[\operatorname{diag}(c) u_{t-} \Delta \bar{Q}_{t}+I\right]^{-1},
$$

with initial condition $\Theta(\tau-, \tau)=I$. Since the coefficients are bounded there exists a unique solution and as it can be checked with the aid of the Ito formula this solution satisfies the relation

$$
\Theta(t, \tau) \Phi^{u}(t, \tau)=I .
$$

Therefore, the matrix $\left.\Phi^{u^{*}}(t, \tau)\right)$ is always invertible and its inverse satisfies the linear differential equation with bounded coefficients, thus it is square integrable and moreover has all moment bounded (for details see Lemma 3.1 in [16].)

By using the relation

$$
\Phi^{u^{*}}(s, t)=\Phi^{u^{*}}(s, 0)\left(\Phi^{u^{*}}(t, 0)\right)^{-1}=\Phi^{u^{*}}(s, 0) \Theta(t, 0)
$$

we obtain

$$
\begin{aligned}
& G_{t}=\int_{t}^{T}\left(\Phi^{u^{*}}(s, t)\right)^{*}\left[f_{0}\left(u_{s}^{*}\right)-k c u_{s}^{*}\right] d s= \\
& \Theta^{*}(t, 0) G_{0}-\int_{0}^{t} \Theta^{*}(t, s)\left[f_{0}\left(u_{s}^{*}-k c u_{s}^{*}\right] d s,\right.
\end{aligned}
$$

where

$$
G_{0}=\int_{0}^{T}\left(\Phi^{u^{*}}(s, 0)\right)^{*}\left[f_{0}\left(u_{s}^{*}\right)-k c u_{s}^{*}\right] d s .
$$

Taking into account (26) we yield

$$
\psi_{t}=\bar{E}\left(G_{t} \mid \mathcal{F}_{t}^{N}\right)=\Theta^{*}(t, 0) \bar{E}\left(G_{0} \mid \mathcal{F}_{t}^{N}\right)-\int_{0}^{t} \Theta^{*}(t, s)\left[f_{0}\left(u_{s}^{*}\right)-k c u_{s}^{*}\right] d s
$$

where the martingale $\bar{E}\left(G_{0} \mid \mathcal{F}_{t}^{N}\right)$ admits the representation

$$
\bar{E}\left(G_{0} \mid \mathcal{F}_{t}^{N}\right)=\bar{E}\left(G_{0}\right)+\int_{0}^{t} \phi_{s} d \bar{Q}_{s}
$$

with some predictable square integrable process $\phi_{t}[20]$. Then the equation (23) follows from (28) by applying the Ito formula. 
Proof of Theorem 4: For a fixed $u \in U, \tau$ and $\varepsilon$, such that $0 \leq \tau \leq \tau+\varepsilon \leq T$, and $A \in \mathcal{F}_{\tau}^{N}$ define a strong variation $u(\cdot)$ of the optimal control $u^{*}$ by putting

$$
u(t, \omega)=\left\{\begin{array}{l}
u, \quad \text { if } \quad(t, \omega) \in[\tau, \tau+\varepsilon] \times A, \\
u^{*}(t, \omega), \quad \text { otherwise }
\end{array}\right.
$$

Then

$$
\begin{gathered}
J\left[u^{*}(\cdot)\right]-J[u(\cdot)]= \\
\bar{E}\left[\int_{\tau}^{\tau+\varepsilon}<f_{0}\left(u_{s}^{*}\right)-k c u_{s}^{*}, \Phi^{u^{*}}(s, \tau) q_{\tau}^{u^{*}}>-<f_{0}\left(u_{s}\right)-k c u_{s}, \Phi^{u}(s, \tau) q_{\tau}^{u^{*}}>+\right. \\
\left.\int_{\tau+\varepsilon}^{T}<f_{0}\left(u_{s}^{*}\right)-k c u_{s}^{*}, \Phi^{u^{*}}(s, \tau+\varepsilon)\left[\Phi^{u^{*}}(\tau+\varepsilon, \tau)-\Phi^{u}(\tau+\varepsilon, \tau)\right] q_{\tau}^{u^{*}}>d s\right]= \\
\bar{E}\left[\int_{\tau}^{\tau+\varepsilon}<f_{0}\left(u_{s}^{*}\right)-k c u_{s}^{*}, \Phi^{u^{*}}(s, \tau) q_{\tau}^{u^{*}}>-<f_{0}\left(u_{s}\right)-k c u_{s}, \Phi^{u}(s, \tau) q_{\tau}^{u^{*}}>d s\right]+ \\
\bar{E}<\psi_{\tau+\varepsilon},\left[\Phi^{u^{*}}(\tau+\varepsilon, \tau)-\Phi^{u}(\tau+\varepsilon, \tau)\right] q_{\tau}^{u^{*}}>.
\end{gathered}
$$

Then by using the estimations

$$
\bar{E}\left[\left\|\Phi^{u}(s, \tau)-I\right\|\right]^{2} \leq L \varepsilon,
$$

which are valid for $s \in[\tau, \tau+\varepsilon]$, we obtain that first integral term in (29) after dividing by $\varepsilon>0$ and letting $\varepsilon \rightarrow 0$ gives the term

$$
\bar{E}\left[<f_{0}\left(u_{\tau}^{*}\right)-k c u_{\tau}^{*}-f_{0}(u)+k c u, q_{\tau}^{u^{*}}>\right] .
$$

In order to estimate the second term in (29) we use the representation of $\psi$ and $\Phi$ by corresponding differential equations and the Ito formula. Since $\Phi_{s}^{u^{*}}=\Phi_{s}^{u}$ for $s \leq \tau$ we obtain

$$
\begin{gathered}
\bar{E}<\psi_{\tau+\varepsilon},\left[\Phi^{u^{*}}(\tau+\varepsilon, \tau)-\Phi^{u}(\tau+\varepsilon, \tau)\right] q_{\tau}^{u^{*}}>= \\
\bar{E}\left[\int_{\tau}^{\tau+\varepsilon}<d \psi_{s},\left[\Phi_{s-}^{u^{*}}-\Phi_{s-}^{u}\right]>+\int_{\tau}^{\tau+\varepsilon}<\psi_{s},\left[d \Phi_{s}^{u^{*}}-d \Phi_{s}^{u}\right]>\right]
\end{gathered}
$$

All integrals over $d \bar{Q}_{s}$ in (30) have zero expectation. The rest of the first integral over $d t$ has a form $\int_{\tau}^{\tau+\varepsilon} \alpha_{s}\left[\Phi_{s-}^{u^{*}}-\Phi_{s-}^{u}\right] d s$, therefore, after dividing by $\varepsilon>0$ and letting $\varepsilon \rightarrow 0$ as above, we obtain the limit $\bar{E}\left\{\alpha_{\tau}\left[\Phi_{\tau-}^{u^{*}}-\Phi_{\tau-}^{u}\right]\right\}=0$. The same operation to be applied to the second integral in (30) gives

$$
\bar{E}<\psi_{\tau},\left[A\left(\tau, u_{\tau}^{*}\right)-A\left(\tau, u_{\tau}\right)\right] q_{\tau}^{u^{*}}>.
$$

INRIA 
Since

$$
\lim _{\varepsilon \rightarrow 0} \frac{J\left[u^{*}(\cdot)\right]-J[u(\cdot)]}{\varepsilon} \geq 0
$$

we obtain finally,

$$
\begin{aligned}
\bar{E}[ & <f_{0}\left(u_{\tau}^{*}\right)-k c u_{\tau}^{*}-f_{0}(u)+k c u, q_{\tau}^{u^{*}}>+ \\
& \left.<\psi_{\tau},\left[A\left(\tau, u_{\tau}^{*}\right)-A(\tau, u)\right] q_{\tau}^{u^{*}}>\right] \geq 0
\end{aligned}
$$

Thereby,

$$
\bar{E}\left[H\left(\psi_{\tau}, q_{\tau}^{u^{*}}, u_{\tau}^{*}, \tau\right)-H\left(\psi_{\tau}, q_{\tau}^{u^{*}}, u, \tau\right)\right] \geq 0
$$

for all $u \in U, \tau \in[0, T]$ and $A \in \mathcal{F}_{\tau}^{N}$. That is the optimal control satisfies the maximum principle (24).

Remark 4 If matrix-valued function $A$ does not depend on $u$ and function $f_{0}$ satisfy (8), then there exists a unique control

$$
u_{t}^{*}=\underset{U}{\operatorname{argmax}}<f_{0}(u)-k c u, q_{t}^{u}>,
$$

where $q_{t}^{u}$ is the corresponding solution of (20). Moreover one can immediately obtain the corresponding solution for the original problem statement, since

$$
\underset{U}{\operatorname{argmax}}<f_{0}(u)-k c u, q_{t}^{u}>=\underset{U}{\operatorname{argmax}}<f_{0}(u)-k c u, \pi_{t}>.
$$

If the function $f_{0}$ is strictly convex, then this control will be piece-wise deterministic optimal, since the number of jumps in each interval is finite almost surely and between the jumps the control is a continuous function of the current state $\pi_{t}$.

Remark 5 Even though the control $u_{t}^{*}$ satisfies the stochastic maximum principle, we cannot conclude for sure that it is an optimal control. The existence theorem is still missing. Therefore, we can say that this control is very likely to be optimal. In the next section, via numerical experiments, we shall provide yet another indirect confirmation of optimality of the constructed control.

\section{Numerical example and comparison with AIMD scheme}

Let us consider a Gilbert type path model with two states. Transitions between two states are governed by a generator

$$
A^{*}=\left(\begin{array}{cc}
-\lambda & \lambda \\
\mu & -\mu
\end{array}\right) .
$$

The losses or ECN marks arrive according to the counting process $N_{t}$ with intensities $c_{1}\left(c_{2}\right)$ in State 1(2). We suppose that $c_{1}<c_{2}$. Thus, State 1 corresponds to a "good" state of the path and State 2 corresponds to a "bad" state of the path, respectively. We can consider

$\mathrm{RR} \mathrm{n}^{\circ} 5239$ 
only one equation for random variable $\pi_{t}^{1}=E\left(I\left\{X_{t}=1\right\} \mid \mathcal{F}_{t}^{N}\right)$ which has the following form

$$
d \pi_{t}^{1}=\left[-\lambda \pi_{t}^{1}+\left(1-\pi_{t}^{1}\right) \mu+u_{t} \pi_{t}^{1}\left(1-\pi_{t}^{1}\right)\left(c_{2}-c_{1}\right)\right] d t-\frac{\pi_{t}^{1}\left(1-\pi_{t}^{1}\right)\left(c_{2}-c_{1}\right)}{\pi_{t}^{1} c_{1}+\pi_{t}^{2} c_{2}} d N_{t} .
$$

Therefore, between the jumps an application of control $u_{t}>0$ makes conditional probability $\pi^{1}$ to increase steadily, and at the jumps this probability abruptly decreases.

We take the following standard utility function

$$
f_{0}(u, x)=-\frac{a^{2}}{R T T^{2}(x) u} .
$$

This utility function corresponds to the AIMD congestion avoidance mechanism in the case of small losses [22]. We would like to note that in the above utility function the Round Trip Time (RTT) parameter depends on the unobservable state of the path.

The optimal control, which maximizes $<f_{0}(u)-k c u, \pi_{t}>$, has the following form

$$
u_{\text {opt }}\left(\pi_{t}\right)=\frac{a}{\sqrt{k}} \sqrt{\frac{\frac{\pi_{t}^{1}}{R T T^{2}(1)}+\frac{1-\pi_{t}^{1}}{R T T^{2}(2)}}{c_{1} \pi_{t}^{1}+c_{2}\left(1-\pi_{t}^{1}\right)}} .
$$

If $R T T(1)=R T T(2)=R T T, \quad k=1, \quad c^{1}=0, \quad c^{2}=c$, we obtain

$$
u_{o p t}\left(\pi_{t}\right)=\frac{a}{R T T \sqrt{c\left(1-\pi_{t}^{1}\right)}}=\frac{a}{R T T \sqrt{\hat{p}_{t}}}
$$

where $\hat{p}_{t}$ is a packet loss probability conditioned on the observed history. It is very interesting that this formula coincides with the one giving the average transmission rate of TCP New Reno over Markovian path where the probability of loss replaced by its conditioned version. Thus, we have got a version of the famous "square root" formula [24].

To perform the numerical experiments, one needs to substitute (32) into (31) and solve this differential equation numerically. It is important to note that one must solve the differential equation and generate the counting process $N_{t}^{u}$ simultaneously, as $N_{t}^{u}$ depends on the sending rate $u$ and the state of the path. Some examples of numerical solutions are presented in Figures 1-4. In particular, one can clearly see from Figure 4 that the performance of the optimal control is more "conservative" than that of AIMD. The following set of parameters was used for the numerical analysis:

$$
\begin{gathered}
\lambda=\mu=2, \quad R T T(1)=R T T(2)=0.5, \quad c_{1}=0.2, \quad c_{2}=1.0, \\
T=10, \quad a=1, \quad k=1 .
\end{gathered}
$$

The evolution of AIMD is modeled by the standard stochastic differential equation

$$
d u_{t}=\frac{d t}{R T T\left(X_{t}^{u}\right)}-\frac{u_{t-}}{2} d N_{t}^{u},
$$




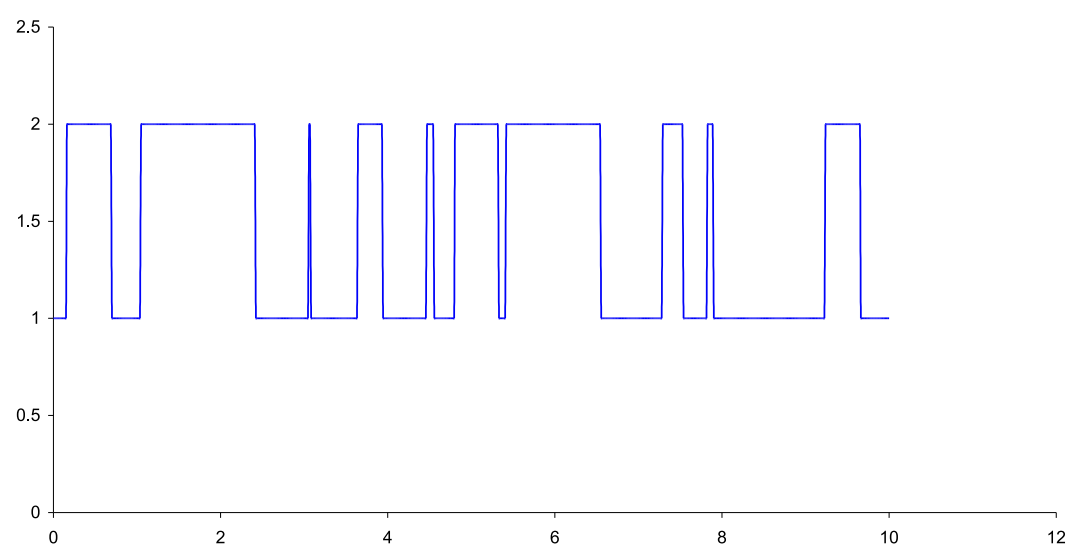

Figure 1: The state of Markov process $X_{t}$.

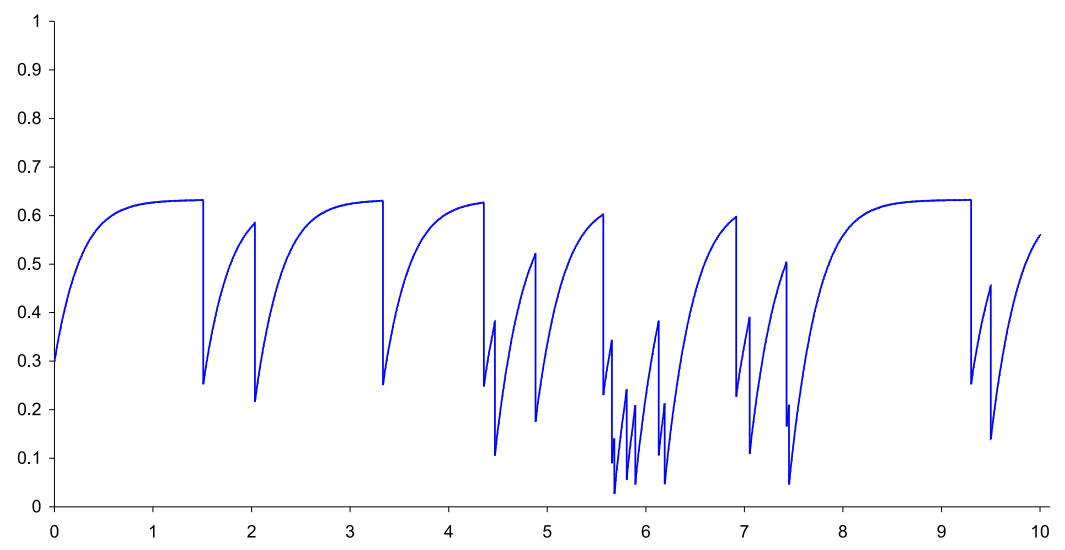

Figure 2: Conditional probability $\pi_{t}^{1}$.

with the initial condition $u_{0}=u_{\text {opt }}\left(\pi_{0}\right)$ in order to remove the effect of transient behavior. In (34) the counting process $N_{t}^{u}$ is generated the same way as for the optimal control.

We would like to note that by changing the parameter $k$, it is easy to tune the degree of "conservativeness" of the control. For instance, if we increase the cost of losses (marks) from 1 to 4 , the average value of the transmission rate decreases and the control becomes nearly constant (see Figure 5).

$\mathrm{RR} \mathrm{n}^{\circ} 5239$ 


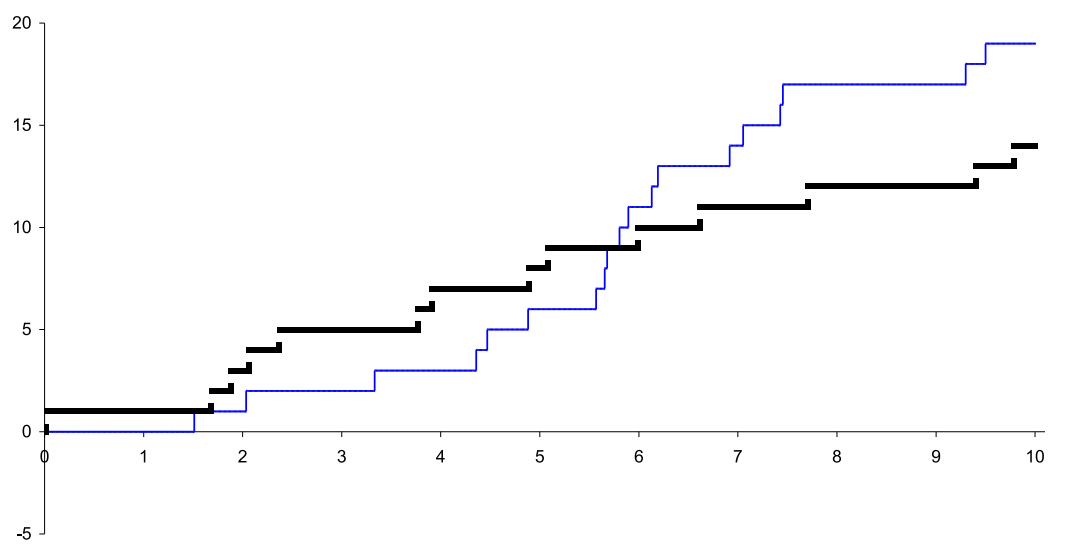

Figure 3: Counting process $N_{t}^{u}$ for the optimal control and the sub-optimal AIMD control.

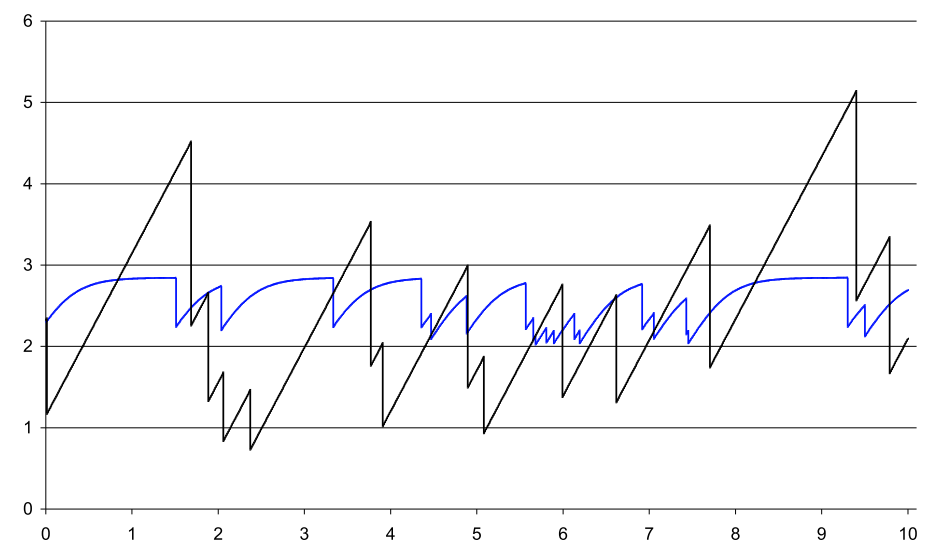

Figure 4: The evolution of the optimal and AIMD controls.

And on contrary, the knowledge about the aprior information about the path characteristics, allows the optimal control increase the sending rate much faster than AIMD and at the same time to be enough prudent to avoid overshots. For instance, if it is known that the path is most of the time in a good state, e.g., $\lambda=2, \mu=6$ and $c_{1}=0.1$, the optimal control really takes an advantage of this aprior information (see Figure 6). The advantage

INRIA 


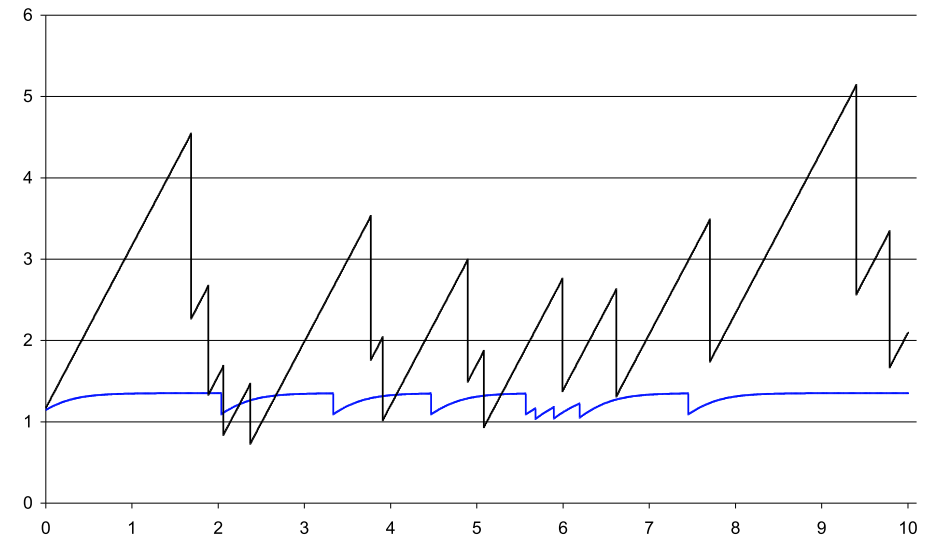

Figure 5: The evolution of the optimal and AIMD controls $(k=4)$.

of the optimal control becomes even more pronounced when RTT changes significantly with the change of the underlying Markov chain (see Figure 7).

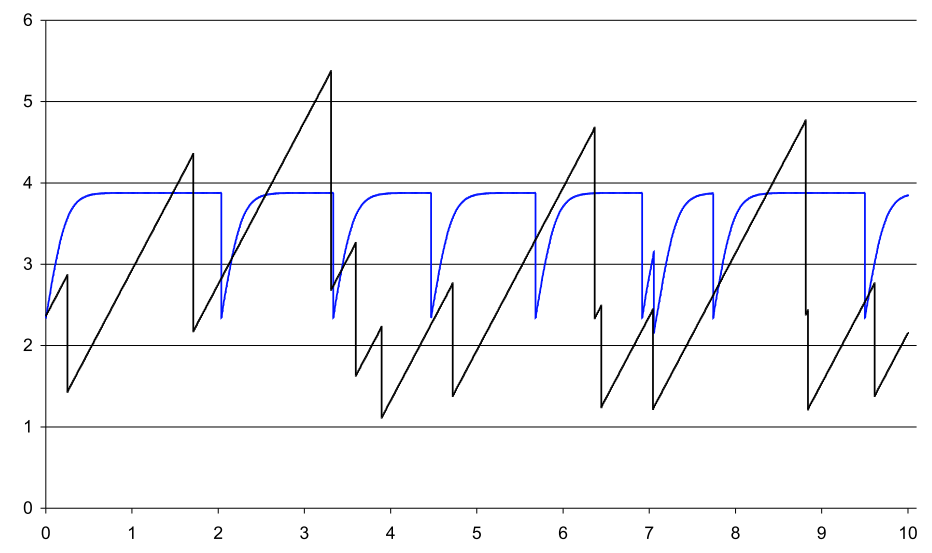

Figure 6: The effect of aprior information on the control evolution.

One can also use another approach for numerical experiments which is based on Girsanov's transformation. In particular, this method is more computationally efficient, since one needs to generate only a homogeneous Poisson process. Furthermore, using this method 


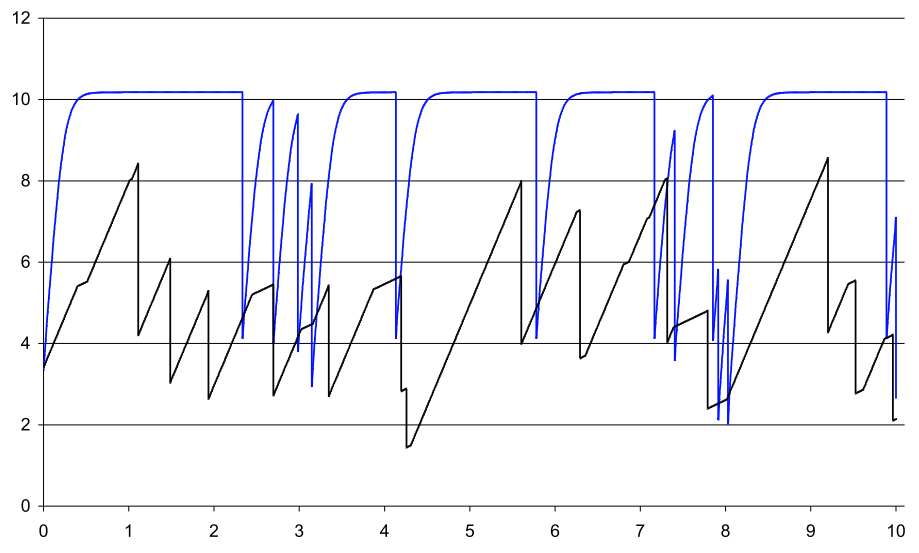

Figure 7: The evolution of the optimal and AIMD controls $(R T T(1)=0.2, R T T(2)=1.0)$.

it is more easy to compare the average values of the control and its variance for the optimal scheme and the sub-optimal AIMD. To calculate the average characteristics, we generate 100 trajectories. This results in the following values for the average performance criterion and sending rates:

$$
\begin{array}{cl}
\bar{J}\left[u_{o p t}\right]=-2.86, & \bar{J}\left[u_{A I M D}\right]=-3.06, \\
\bar{u}_{o p t}=2.43, & \bar{u}_{A I M D}=2.32 .
\end{array}
$$

Even though the differences between the respective average characteristics is less than $10 \%$, the difference between the standard deviations is quite impressive (486\%):

$$
\sigma\left(u_{\text {opt }}\right)=0.76, \quad \sigma\left(u_{A I M D}\right)=4.45 .
$$

In other words, the optimal control produces much smaller variations of the sending rate and at the same time achieves the sending rate greater than that of AIMD. This nice property can be utilized in the context of the TCP-friendly protocols [13]. In Figures 8 and 9 we plot the average sending rates and their standard deviations along the time interval $[0, T]$ for the optimal and AIMD controls, respectively.

\section{Conclusions and future research}

The presented model allows us to answer several fundamental questions about the evolution of TCP sending rate. In particular, we show that for a Markovian path the optimal sending rate evolution is indeed piece-wise deterministic as it is the case in the current TCP implementation. However, we also show that the optimal increase is not linear and the 


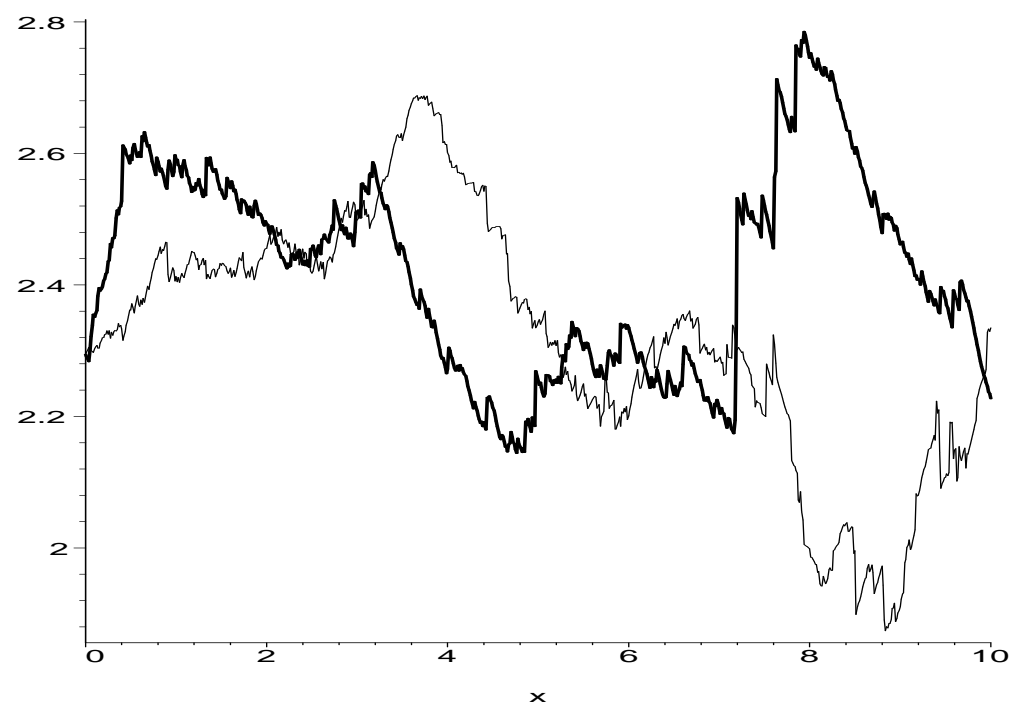

Figure 8: The averages along $[0, T]$ of the optimal (thick line) and AIMD (thin line) controls.

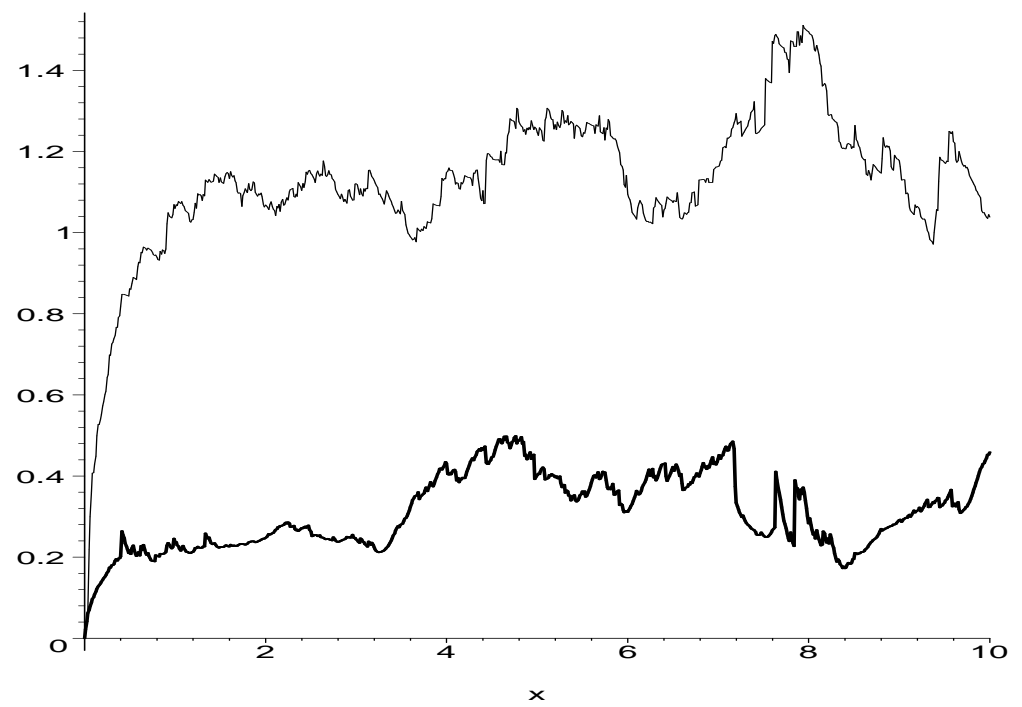

Figure 9: The standard deviations along $[0, T]$ of the optimal (thick line) and AIMD (thin line) controls.

$\mathrm{RR} \mathrm{n}^{\circ} 5239$ 
optimal multiplicative decrease is not proportional to the instantaneous sending rate. The optimal control take advantage from aprior information about the path characteristics. In particular, this allows congestion control to achieve much smaller variance of the sending rate evolution and at the same time to gain in the average throughput. The latter property of the optimal control can be exploited by the TCP-friendly rate control protocols. It seems that our results could be very helpful in the ensuing research directions:

1. the case of control dependent generators $A$, here a typical problem is the estimation of the buffer state and the flow control in the presence of unknown parasitic flows;

2. identification of the loss types: congestion versus transmission losses; in particular, in the context of wireless networks;

3. inclusion into the observation framework another parameters correlated with the state of the path, such as the RTT parameter.

\section{References}

[1] M. Allman, V. Paxson and W. Stevens, TCP congestion control, RFC 2581, April 1999, available at http://www.ietf .org/rfc/rfc2581.txt.

[2] E. Altman, K. Avrachenkov and C. Barakat, A stochastic model of TCP/IP with stationary random losses, ACM SIGCOMM'00, Computer Communications Review, 30(4), (2000) 231-242.

[3] E. Altman, K. Avrachenkov and C. Barakat, TCP in presence of bursty losses, Performance Evaluation, 42, (2000), 129-147.

[4] E. Altman, K.E. Avrachenkov, C. Barakat and P. Dube, TCP over a multi-state Markovian path, in Performance and QoS of Next Generation Networking, eds. K. Goto, T. Hasegawa, H. Takagi and Y. Takahashi, Springer Verlag, (2000), 103-122.

[5] S. Athuraliya and S. Low, Optimization Flow Control with Newton-Like Algorithm, Telecommunication Systems, 15(3/4), (2000), 345-358.

[6] C. Barakat, TCP/IP modeling and validation, IEEE Network, (2001), May/June, $38-47$.

[7] P.M. Brémaud, Point Processes and Queues, Springer Verlag, Berlin, 1981.

[8] M.H.A. Davis, Markov Models and Optimization, Chapman \& Hall, London, 1993.

[9] G.B. Di Masi and P.I. Kitsul, Backward representation for nonstationary Markov processes with finite state space, Systems \& Control Lett. 22 (1994) 445-450.

[10] R.J. Elliott, L.Aggoun and J.B. Moore, Hidden Markov Models. Estimation and Control, Springer Verlag, New York, 1995.

INRIA 
[11] W.H. Fleming, R.W. Rishel, Deterministic and Stochastic Optimal Control, SpringerVerlag, Berlin, 1975.

[12] S. Floyd and V. Jacobson, Random Early Detection Gateways for Congestion Avoidance, IEEE/ACM Transactions on Networking, 1(4), (1993), 397-413.

[13] S. Floyd, M. Handley, J. Padhye, and J. Widmer, Equation-Based Congestion Control for Unicast Applications, In Proceedings ACM SIGCOMM, August 2000.

[14] E.N. Gilbert, Capacity of a burst-noise channel, Bell Systems Technical Journal, 39, (1960), 1253-1266.

[15] V. Jacobson, Congestion avoidance and control, ACM SIGCOMM'88, August 1988.

[16] Yu.M. Kabanov, On the Pontriagin maximum principle for SDEs with Poissontype driving noise, in: Stochastic and Control of Stochastic Processes, Moscow (1995/1996), World Sci. Publishing, River Edge, New York, 1997, 173-189.

[17] F.P. Kelly, Mathematical modelling of the Internet, in: Mathematics Unlimited 2001 and Beyond, eds. B. Engquist and W. Schmid. Springer Verlag, Berlin, (2001), $685-702$.

[18] F.P. Kelly, A. Maulloo and D. Tan, Rate control in communication networks: shadow prices, proportional fairness and stability, Journal of the Operational Research Society, 49, (1998), 237-252.

[19] S. Kunniyur and R. Srikant, End-to-end congestion control: Utility functions, random losses and ECN marks, in Proc. IEEE INFOCOM'00, Tel Aviv, Israel, (2000), also to appear in IEEE/ACM Transactions on Networking.

[20] R.Sh. Liptser, A.N. Shiryayev, Statistics of Random Processes, Springer-Verlag, Berlin, 1977.

[21] S.H. Low and D.E. Lapsley, Optimization Flow Control, I: Basic Algorithm and Convergence, IEEE/ACM Transactions on Networking, 7(6), (1999), 861-874.

[22] S.H. Low, F. Paganini and J.C. Doyle, Internet Congestion Control, IEEE Control Systems Magazine, 22(1), (2002), 28-43.

[23] L. Massoulié and J. Roberts, Bandwidth sharing: objectives and algorithms, IEEE/ACM Transactions on Networking, 10(3), (2002), 320-328.

[24] M. Mathis, J. Semke, J. Mahdavi, T. Ott, The Macroscopic Behavior of the TCP Congestion Avoidance Algorithm, Computer Communication Review, 27, (1997), n. 3 , July.

[25] B.M. Miller and W.J. Runggaldier, Optimization of observations: a stochastic control approach, SIAM J. Control Optim., 35(3), (1997), 1030-1052. 
[26] B.M. Miller and W.J. Runggaldier, Kalman filtering for linear systems with coefficients driven by a hidden Markov jump process, Systems \& Control Letters, 31, (1997), 93-102.

[27] K. Ramakrishnan and R. Jain, A binary feedback scheme for congestion avoidance in computer networks with connectionless network layer, ACM SIGCOMM'88, August 1988, also in ACM Transactions on Computer Systems, 8(2), (1990), 158-181.

[28] K. Ramakrishnan, S. Floyd and D. Black, The Addition of Explicit Congestion Notification (ECN) to IP, $R F C$ 3168, September 2001, available at http://www .ietf .org/rfc/rfc3168.txt.

[29] V. Dumas, F. Guillemin and P. Robert, A Markovian analysis of AIMD algorithms, Advances in Applied Probability, 34(1), (2002), 85-111.

[30] S. Tang and X. Li, Necessary conditions for optimal control of stochastic systems with random jumps, SIAM J. Control Optim., 32(5), (1994), 1447-1475.

[31] E. Wang and B. Hajek, Stochastic Processes in Engineering Systems, Springer-Verlag, Berlin, Heidelberg, New-York, 1985. 


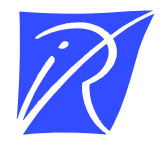

\section{Unité de recherche INRIA Sophia Antipolis 2004, route des Lucioles - BP 93 - 06902 Sophia Antipolis Cedex (France)}

Unité de recherche INRIA Futurs : Parc Club Orsay Université - ZAC des Vignes 4, rue Jacques Monod - 91893 ORSAY Cedex (France)

Unité de recherche INRIA Lorraine : LORIA, Technopôle de Nancy-Brabois - Campus scientifique 615, rue du Jardin Botanique - BP 101 - 54602 Villers-lès-Nancy Cedex (France)

Unité de recherche INRIA Rennes : IRISA, Campus universitaire de Beaulieu - 35042 Rennes Cedex (France)

Unité de recherche INRIA Rhône-Alpes : 655, avenue de l'Europe - 38334 Montbonnot Saint-Ismier (France)

Unité de recherche INRIA Rocquencourt : Domaine de Voluceau - Rocquencourt - BP 105 - 78153 Le Chesnay Cedex (France)

INRIA - Domaine de Voluceau - Rocquencourt, BP 105 - 78153 Le Chesnay Cedex (France)

http://www.inria.fr

ISSN 0249-6399 